Chapter I

Review Papers

Harlow Shapley 


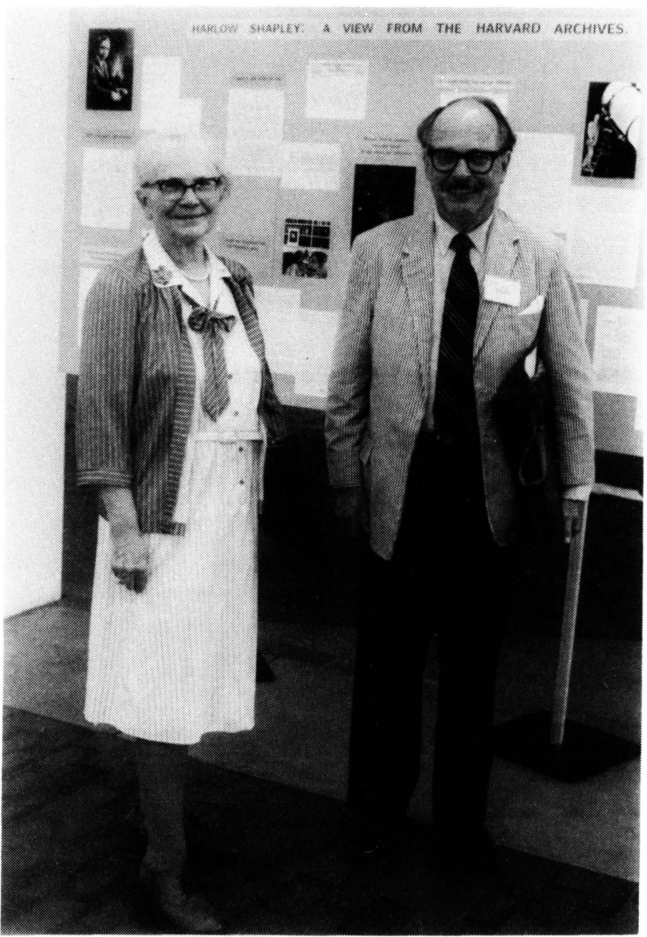

Helen Sawyer Hogg and Willis Shapley remembering

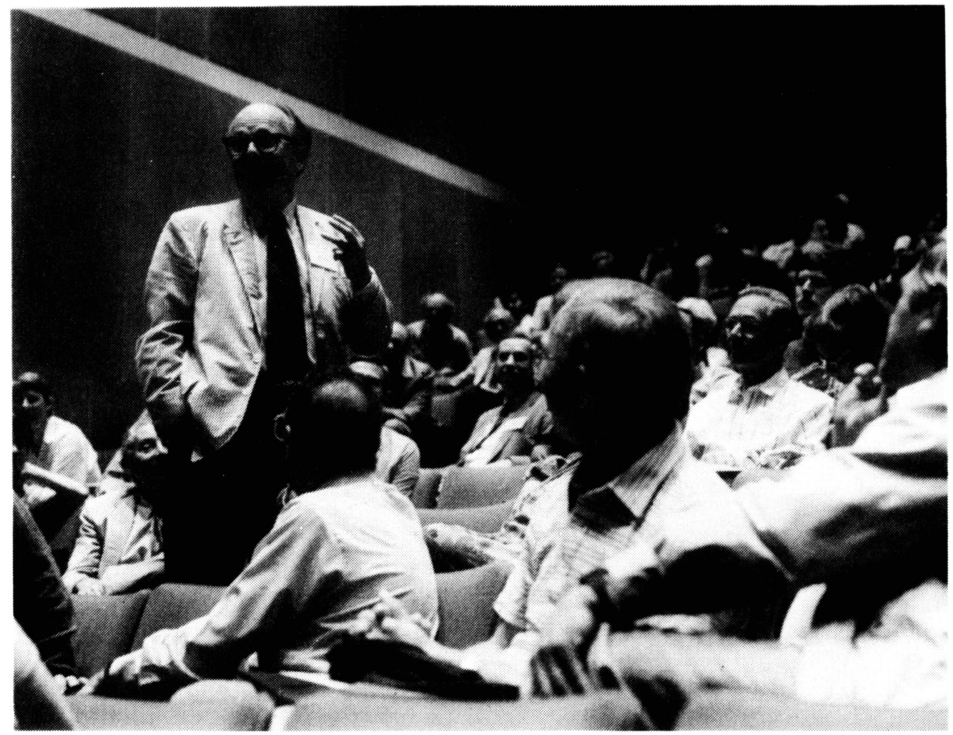

Willis Shapley fielding questions on his father 
The attempt to make three-dimensional sense of the Milky Way goes back to a most unlikely origin: the English antiquary of the early eighteenth century, William Stukeley, remembered today for associating the Druids with Stonehenge. Stukeley came from Lincolnshire and so was a fellow-countryman of Isaac Newton, and as a result he was privileged to talk with the great man from time to time. In his Memoirs of Newton Stukeley records one conversation they had in about 1720, in which Stukeley proposed that the Sun and the brightest stars of the night sky make up what we today would term a globular cluster, and this cluster is surrounded by a gap, outside of which lie the small stars of the Milky Way in the form of a flattened ring.

Stukeley's remarkable suggestion was recorded only in his manuscript memoirs, and had no effect on the subsequent history of astronomy. It chanced that the very same model was proposed a century later by John Herschel, who in 1833 in his A treatise on astronomy compared the appearance of "our own sidereal firmament and milky way" to Saturn and its ring. Herschel afterwards took his $20 \mathrm{ft}$ reflector to South Africa and between 1834 and 1838 subjected the southern skies to their first close scrutiny. He decided that the structure of the Galaxy is immeasurably more complex than his simple Saturn model had allowed; and that in fact the Sun and the brighter stars occupy a sparsely-populated region surrounded by a dense and complex ring of stars whose more distant windings extend out to the limits of telescopic vision and beyond. Herschel's unique status as the only astronomer in history systematically to examine the whole of the sky, both northern and southern hemispheres, with a major telescope, discouraged lesser mortals from theorising about the Milky Way for the next half-century. Theorising resumed when Jacobus Kapteyn realised that, as an astronomer without a telescope, he could nevertheless get access to a wealth of new information if he offered his services in measuring the positions of stars on David Gill's Cape Photographic Durchmusterung then in progress, and this he duly did with the help of teams of convicts from Groningen State Prison. Right up to his death in 1922 Kapteyn worked to determine the structure of the Galaxy. He was of course, like his predecessors, working outwards in all directions from the location of the observer in the solar system; and it could hardly be otherwise. Kapteyn was alert to the threat posed to his analysis by possible obscuration in the plane of the Galaxy, but the reddening of light that he thought would reveal such obscuration was not in fact observed. As a 
result, he believed that the whole of the Galaxy was accessible to his investigations when in fact he could see only our neighbourhood; and the more remote stars in the galactic plane seemed fainter and more thinly scattered than was in fact the case, so that the Galaxy seemed to be only a few thousand light years in size and the solar system fairly central.

A crucial clue lay hidden in the verbose prose of John Herschel's volume reporting his Cape observations. He speaks (p. 136) of "the extraordinary display of fine ... globular clusters" in the general direction of Sagittarius. "Here, in a circular space of $18^{\circ}$ in radius, we find collected no less than thirty of these beautiful and exquisite objects.... are we to connect it with the very peculiar structure of the Milky Way in this particular part of its course, which is here unlike in its constitution to any other portion of that zone, and which passes diametrically across the circular area in question". The fact that the great majority of globular clusters lie in one half of the sky was remarked on in 1909 by the Swedish astronomer Karl Bohlin, and he proposed that the globular clusters are packed together close to the centre of the Galaxy and clustered symmetrically around it (so that the Sun is eccentric in position); and that this "cluster of clusters" is surrounded by the star clouds of the Milky Way together with the Sun and other isolated stars.

Bohlin went on to propose that the entire Galaxy is a former planetary nebula, the material at whose poles has dispersed to form spiral nebulae as the great planetary nebula has rotated. The material in the equatorial zone of the planetary nebula is still in position, though it has condensed to form the star clouds of the Milky Way.

Bohlin's bizarre paper, tucked away in the proceedings of a Swedish journal devoted to general science, and the work of an author as eccentric as the location he assigned to the Sun, carried little weight; and Bohlin's insight into the peculiar distribution of the globular clusters had little effect. It was however known some years later to the rising young star of American astronomy, Harlow Shapley. Shapley had been born in 1885, and after some experience as a teenage reporter on a newspaper had enrolled in 1907 at the University of Missouri, intending to study in the University's School of Journalism. Finding the school was not to open for another year, he looked round for something else to study, and hit on astronomy. When Shapley was in his third year as a student, Frederick H. Seares of the Laws Observatory offered him a teaching assistantship, and was sufficiently impressed by the young man later to recommend him to Princeton for a fellowship in astronomy. There Shapley became a pupil of Henry Norris Russell, a legendary orator with wide-ranging interests in astronomy and astrophysics. Russell put Shapley to work on eclipsing binaries, and in his doctoral dissertation Shapley discussed the orbits of ninety such binaries where only a handful had been studied previously. He also showed that if Delta Cephei were to be an eclipsing binary, as commonly believed, the two components would have to fall inside each other; instead he proposed that it was a single, pulsating star.

Meanwhile Seares had moved to Mount Wilson, where he told George Ellery Hale of Shapley's ability. Hale interviewed him and appointed him to the Mount Wilson staff. Solon I. Bailey of Harvard advised Shapley to use the 60 -inch telescope to study variables in globular clusters. Bailey himself had already 
detected a number of Cepheids in globular clusters, and Henrietta Leavitt had earlier identified many Cepheids in photographs of the Small Magellanic Cloud. As early as 1908 Miss Leavitt had noted that the longer the period of the star, the brighter it appeared on her photographs, and therefore the more luminous it was. In a further paper in 1912 she set out her results graphically, and pointed out that if her photographic magnitude scale could be calibrated for absolute magnitude, the distance of the Cloud could be determined.

Shapley's discovery that Cepheids were not binary stars, whose appearance would depend upon the chance geometrical configuration relative to the observer, but pulsating stars displaying the results of intrinsic physical processes, must have brought home to him the plausibility of using Cepheids as distance indicators. Before long he was being allowed substantial periods of time on the 60 -inch for his own research on variables in clusters, and he managed to calibrate Cepheids by means of an ingenious and not wholly convincing statistical argument. In 1915 he noted that globular clusters are largely concentrated into one hemisphere in galactic longitude, and he remarks on Bohlin's theory -- though we must remember that for Bohlin the globulars are densely concentrated at the centre of the Galaxy, and are themselves surrounded at a great distance by the ring of the Milky Way stars. But for Shapley in 1915 the accepted size of the Galaxy -- a few thousand light years in diameter -- was so small compared to the distances he was deriving for globular clusters that the jigsaw refused to fall into place. It seemed that globular clusters were "very distant systems, distinct from our Galaxy and perhaps not greatly inferior to it in size".

In the winter of $1917 / 18$ came the breakthrough, as Shapley brought himself to reject the accepted size of the Galaxy. As he wrote to Eddington at the time, "The globular clusters outline the sidereal system." His conclusion was that we are some 60,000 light years away from the centre of the system of globulars, and therefore the same distance from the centre of the Galaxy, whose diameter he now estimated at 300,000 light years. This was a dramatic conclusion, based on an immense amount of detailed labour, but also on some questionable steps, as with the calibration of the Cepheids. Shapley was at this time acquiring an unfortunate reputation for irresponsible speculation. In 1919, for example, he downgraded the spiral nebulae to being insubstantial, gaseous bodies being driven off by radiation pressure from our Galaxy. Both Hale and Russell wrote to warn him of the risks he was taking with his standing as an astronomer. Russell wrote: "... I am sorry to see you join the company of those who advance theories that are 'startling if true'. There has been a great deal too much of this done in the last few years."

The enormous increase that Shapley proposed in the size of the Galaxy naturally aroused great controversy, for his methods challenged the traditional techniques for working steadily outwards from the solar system towards the more remote regions of the Galaxy. Shapley's big Galaxy also made it almost unthinkable that the innumerable spiral nebulae could be other such galaxies, and so Shapley had become caught up in the age-old problem of the status of the nebulae: were they enormous star systems, 'island universes', -- or were they nearby clouds of chaotic matter? Christopher Wren had taken the former position, Edmond Halley the latter. In the 1860 s the spectroscope had shown that some nebulae are gaseous; but what of the tiny spirals, of which hundreds of thousands appeared on the Lick Observatory photographs? 
Early in the twentieth century three compelling pieces of evidence argued against the spirals being island universes comparable to our Galaxy. First was the 'zone of avoidance': spiral nebulae were dense around the poles of the Galaxy, and avoided the plane of the Galaxy. Surely this must mean that the spirals were related to our Galaxy and not independent galaxies in their own right. Heber Doust Curtis of Lick had found the solution to this difficulty in the photographs he had taken of edge-on spirals, with dark lanes of dust clearly visible. Similar dust in the plane of our Galaxy would conceal from us any spirals that lay in low galactic latitudes.

Secondly there was the new star that had flared up in the Andromeda Nebula in 1885. At one stage the star, S Andromedae, was about one-tenth the brightness of the entire nebula, and so if the nebula was an island universe of millions of stars, this nova must have reached the brightness of hundreds of thousands of stars, and this in only a few hours. No processes known to physics could achieve such sensational results; much more likely that the nebula was a gas cloud that had encountered a passing star, and that the star had flared up as it passed through the cloud. Here again Curtis had found the answer. In 1917, on photographs of other spirals, he had come across further examples of novae; and these were fireworks on a much more modest scale. While Curtis was cautiously examining other photographs of these particular spirals, George W. Ritchey of Mount Wilson discovered a nova that was still visible. This sent a number of astronomers (including Shapley) hurrying to compare photographs of spirals, and several more were found. All were markedly less dramatic than $S$ Andromedae and the $Z$ Centauri of 1895, which began to be recognised as exceptional. As Curtis suggested in 1921, "a division into two magnitude classes is not impossible." If the novae recently discovered were comparable to galactic novae, then the spirals could well be distant enough and large enough to be independent island universes.

The third item of evidence was more recent: the huge recession velocities of spirals measured by Vesto M. Slipher. These were difficult to explain on any theory, but at least the velocities were much larger than those of any known components of our Galaxy.

As a result of all this, Curtis and many of his Lick colleagues were convinced that the spirals were other island universes. But meanwhile, at Mount Wilson, a striking new piece of counter-evidence was at hand: Adriaan van Maanen, a meticulous worker, had used a stereocomparator to compare old and new photographs of $M 101$, and had concluded that the spiral was rotating in only a few thousands of years -- so rapidly that the outlying parts would need to travel impossibly fast if the spiral was a huge island universe. Shapley was van Maanen's friend, and believed him. Curtis did not.

Although it was entirely possible that the spirals might be star systems, or island universes, that were orders of magnitude smaller than our Galaxy, the instinct of astronomers then as always was to pose the question in its simplest terms: was the Galaxy one of the spiral nebulae? If Shapley was correct, and the diameter of the Galaxy is ten times greater than had been thought, and therefore its volume a thousand times greater, the answer was much more likely to be No. And so it is not surprising that when in late 1919 Hale was looking for a topic for the 
annual lecture at the National Academy of Sciences in memory of his philanthropist father, William Ellery Hale, he should think of a debate, between Shapley and a suitable opponent. A few weeks later, in February 1920, Shapley received a telegram inviting him to debate with Heber Curtis of Lick Observatory, 45 minutes each on the scale of the universe.

The invitation could not have come at a more awkward time for Shapley, whose confidence in his ability as an astrophysicist was matched by his confidence in his ability as a future observatory administrator. A year earlier death had at last brought to an end Edward Pickering's 42-year reign as Director of the Harvard College Observatory. The moment he heard the news, Shapley decided he would try to be the next director, and wrote to Russell and Hale to enlist their support.

Both men were aghast at Shapley's naked ambition, and told him so in forthright terms. Russell remarked to Hale that Shapley "would not suffer if he pondered the old fairy tale about the man who got all sorts of good things from a magic fish whose life he had saved, -- until his wife wanted to be Pope!" Yet in fact both Hale and Kapteyn considered Shapley's scientific brilliance made him the best man for the job, although both realised that his youth and lack of experience in the management of staff would make it a risky appointment. Despite their misgivings, both these powerful advocates lobbied Harvard on Shapley's behalf. Hale's second choice was Frank Schlesinger of Allegheny Observatory, then in his late forties and a 'safe' appointment. His third was Russell himself, who would have been an obvious candidate save for one failing: he was a notoriously bad administrator. Harvard dithered, and when Hale's telegram reached Shapley the post had already been left unfilled for a full year. Shapley's own hopes had been encouraged a few weeks earlier when the President of Harvard telegraphed Mount Wilson to know if Shapley had plans to visit the East Coast. On learning that Shapley had no such plans, Harvard had sent one of their Regents to visit Shapley. The Regent knew nothing about science, but was much interested in a AAAS convention that Shapley had organised. The reason was that Harvard had no doubts about Shapley as a scientist, but wanted to reassure themselves as to his abilities as an administrator. Shapley, his ambition fuelled by the visit, wrote to Russell in great excitement, saying "I might say that I am very confident that Harvard is not too big for me and that the thing I could and would do there would be a credit to American astronomy".

Confident that he was under active consideration for the coveted directorship, Shapley read the telegram from Hale with dismay. He judged, correctly, that a delegation from Harvard would come to Washington to see how he performed under fire, and the Academy meeting would be make-or-break. He was then inexperienced as a public speaker, and in fact spoke poorly at the meeting -Russell afterwards expressed the hope that if Shapley came to Harvard as his number two, he would offer a lecture course, for this "cultivates the gift of the gab, which he needs". Curtis was by contrast an excellent lecturer, and in defending the traditional position he had a much easier task. Shapley accordingly set about an exercise in damage limitation. He tried to defuse the encounter by turning it into a partnership rather than a confrontational debate; but here he reckoned without Curtis's Irish ancestry. Curtis relished "a good friendly scrap.... It might be far more interesting both for us and our jury, to shake hands, metaphorically speaking, at the beginning and conclusion of our talks, but use our shillelahs in the 
interim to the best of our ability." Shapley then sought to trivialise the occasion, by reducing the time allotted from 45 to a mere 35 minutes; but after much writing of letters -- happily for historians, the telephone was not then much used -- a compromise of 40 minutes was agreed. This still left him dangerously exposed, so he prepared a presentation so elementary that of the 16 typed pages that Shapley devoted to the topic of the evening, it took him seven to reach the definition of a light-year -- and this to the National Academy of Sciences! Curtis listened to Shapley with dismay, considered abandoning his own closely argued thesis, but decided at the last moment to go ahead.

Shapley's last defensive measure was to persuade Russell that Russell's own ideas would be equally under attack, and to arrange for the chairman to call R ussell to speak first from the floor, to undo the harm that Curtis had done. In fact Russell made so substantial contribution that the question arose as to whether he should appear in the published version as a third contributor.

To analyse the detailed scientific arguments that eventually appeared in the published version would be to misrepresent the actual encounter, as many incautious historians have done in the past. Shapley took as the prime topic, the dimensions of the Galaxy, which he claimed was far larger than had been thought and unlike any other object known to observational astronomy; Curtis was more concerned with comparing the Galaxy to the spiral nebulae. To this extent it is true to say that both men were partly right and partly wrong. Curtis was convinced he had come off best, and this is certainly the case.

But what of Harvard? In writing to President Lowell in March to tell him of Shapley's forthcoming appearance in Washington, Hale began to have misgivings about his earlier support for Shapley, and took the opportunity to hedge his bet by arguing at length the case for yet another candidate, namely Seares, to whom, he said, he would confidently entrust his own observatory if Walter Adams were unavailable. Shapley, he said, was versatile, daring and industrious, and had shown brilliant and unusual qualities in his recent work; but he had not yet reached complete maturity or established his final place. His appointment at Harvard would involve a certain measure of risk. Nevertheless, Hale believed he would be a great success there.

The late introduction of Seares into the discussion was seen at Harvard as reflecting no credit on Hale, and only served to confirm the influential G.R. Agassiz in his support for Shapley. But then came the disaster that Shapley had foreseen: Agassiz attended the Shapley-Curtis debate, and wrote next day to Lowell to say that "Shapley lacks maturity and force, and does not give the impression of being a big enough personality for the position". Russell, who had impressed Agassiz when speaking from the floor at the debate and in private talks afterwards, "besides being more mature, has more balance more force and a broader mental range". And so, before long, Harvard decided to grasp the nettle and offer the directorship to Russell.

In a long and frank letter, Russell turned to Hale for advice. One problem was, "If I don't go to Harvard, who will?" Russell's solution was to have a team, to include a post tailor-made for Shapley: "A second astronomer, younger, and with modern ideas, to be called, to act as the Director's right hand man...." But in the 
end Russell turned the job down.

Harvard were now back to square one. Should they risk appointing Shapley, with all his limitations? A bizarre compromise was now proposed, whereby the distinguished geometer Julian Coolidge should take responsibility for the Observatory, but with Shapley invited to be leader in the scientific work. And so it was that Shapley found himself that November being invited to become, not Director, but "the technical man", with the title of Assistant Professor and Astronomer.

To be expressly denied the directorship, even when Harvard had proved incapable of filling the senior post, must have been a bitter disappointment. Shapley wrote for more information as to how the responsibilities would be divided between Coolidge and himself, but he had enough maturity quickly to realise that the arrangement would be an unworkable compromise, and he soon turned the offer down.

Once again Harvard were back to square one. But now Hale quickly stepped in, and with considerable statesmanship told Lowell that he was prepared to give Shapley a year's leave if he wished to go to Harvard on a trial basis. This gave all parties the opportunities they had been seeking. In April 1921 Shapley took up residence at Harvard; and he must have made a far better impression than expected, for that October he was at last appointed Director. The fisherman's wife had become Pope.

\section{ACKNOWLEDGEMENTS AND BIBLIOGRAPHY}

I am grateful to Harvard University Archives for access to documents relating to the appointment of a successor to Pickering and for permission to quote. My attention was kindly drawn to these documents by Owen Gingerich and the later paragraphs of this paper have greatly benefited as a result.

The text of Shapley's Washington paper, and of the typed slides summarising Curtis's argument, are reprinted with documentation and archival acknowledgements in M. Hoskin, 1976, J. Hist. Astron. 7, 169. This also appears with other relevant material in M. Hoskin, 1982, Stellar Astronomy: Historical Studies, Science History Publications Ltd, Chalfont St Giles, Bucks, UK. On Stukeley, see M. Hoskin, 1985, J. Hist. Astron. 16, 77. On John Herschel, see M. Hoskin, 1987, ibid. 18, 1. On Kapteyn, see E.R. Paul., ibid. 17, 155. On Shapley, see H. Shapley, 1969, Through Rugged Ways to the Stars, Scribner's Sons, New York, and the article by $\mathrm{O}$. Gingerich in Dictionary of Scientific Biography. On the 'Debate' and its background, see R. Berendzen, R. Hart and D. Seeley, 1976, Man Discovers the Galaxies, Neale Watson Academic Publications, New York, and more especially R. Smith, 1982, The Expanding Universe: Astronomy's 'Great Debate' 1900-1931, Cambridge University Press. 